\title{
Aplicación del ABP en grupos grandes en docencia en el Grado en Medicina
}

Ana Lloret ${ }^{a}$ e Isaias Sanmartín ${ }^{b}$

a. Departamento Fisiología. Facultad de Medicina. Universidad de Valencia. ana.lloret@uv.es; b. Facultad de Biotecnología. Universidad Católica de

Valencia. isaias.sanmartin@ucv.es

\begin{abstract}
Resumen
El "Problem Based Learning" (PBL) o "Aprendizaje basado en Problemas" $(A B P)$ es una técnica docente muy utilizada en docencia universitaria aunque todavía no demasiado extendida en la universidad española. No todos los profesores que se atreven a su uso, principalmente por los problemas inherentes a la docencia en grupos grandes de alumnos y de la falta de flexibilidad de los programas docentes de nuestras universidades. En este trabajo comunicamos nuestra experiencia en la aplicación durante 6 años del ABP en grupos grandes y analizamos los resultados tanto a nivel de los alumnos como del profesorado que ha utilizado esta metodología. Los resultados muestran que los alumnos tienen una sensación de aprendizaje muy elevada y que el proceso resulta altamente satisfactorio tanto para alumnos como para los profesores implicados.
\end{abstract}

Keywords: Aprendizaje basado en problemas, casos de estudio.

\section{Introducción}

El Aprendizaje Basado en Problemas (ABP) es una metodología de enseñanza en la cual el aprendizaje del alumno es activo y, lo que es más importante, autodirigido. El ABP se realiza mediante un "caso de estudio" que expone una situación similar a las que se pueden encontrar en la práctica profesional y que plantea un problema. Mediante una serie de preguntas o cuestiones se estimula a los estudiantes para que decidan qué necesitan aprender, dónde y cómo buscarlo y aplicarlo para resolver el problema planteado (Schwartz y cols, 2001). Esta metodología ha mostrado una elevada utilidad para que los alumnos no sólo aprendan "a aprender" y con autonomía, sino que alcancen competencias específicas difíciles de obtener mediante métodos de enseñanza más tradicionales (Albanese y Mitchel, 1993).

El ABP empezó aplicándose en facultades de medicina en Canadá (Barrows y Hamblyn 1980). En la actualidad se ha extendido y aplica a muchas otras disciplinas como enfermería, veterinaria, ciencias biológicas, químicas, física, derecho, económicas, 
ingenierías, etc (Allen y Duch, 1998; Kaufman et al, 1989; Sulaiman, 2010; Newman, 2005), al probarse su gran eficacia formativa (Norman y Schmitdt 2000.

Está pensado para realizarse en pequeños grupos de trabajo bajo la supervisión de un tutor (Aspy, Aspy, y Quimby, 1993; Duch, Groh y Allen, 2001). En este trabajo, hemos utilizado el ABP con el objetivo principal de desarrollar competencias profesionales en estudiantes de físiología del grado en Medicina. Existen diversas variantes en cuanto al desarrollo del método ABP. En nuestro trabajo, hemos aplicado una adaptación de la metodología ABP $4 \times 4$ desarrollada por Prieto y colaboradores en la Universidad de Alcalá de Henares (Prieto et al, 2006).

Con la implementación del nuevo grado en Medicina en el año 2010 y el cambio en el plan de estudios de la Universidad de Valencia, aparece la posibilidad de utilizar nuevas metodologías docentes. Sin embargo, nos encontramos con planes de estudio cerrados en los que es difícil aplicar la transversalidad, así como incorporar técnicas de autoaprendizaje. De forma interesante, se reserva una pequeña parte de la docencia presencial para "grupos tutorizados", en los que el profesor tiene libertad para realizar las actividades formativas con sus alumnos que crea más conveniente. De esta manera, decidimos iniciar actividades ABP siguiendo el ejemplo de los pioneros en nuestro país (Branda, 2001; Font, 2003; Molina, García, Pedraz ycols 2003, Prieto y cols., 2006).

\section{Objetivos}

Las competencias profesionales objetivo de nuestra actividad incluyen la búsqueda de información. Para resolver el caso de estudio planteado pueden utilizarse desde artículos originales de investigación en lengua inglesa hasta libros de Fisiología general o información obtenida de la Internet (que los alumnos debe valorar en cuanto a su confiabilidad o exactitud).

Otro objetivo consiste en el estudio y asimilación de la materia propuesta, así como en la preparación y exposición de una presentación oral y una subsiguiente discusión científica. Se pretende valorar el dominio de tecnologías de la información y comunicación.

Se pretende además que los alumnos cambien su actitud hacia sus propios procesos de aprendizaje, desarrollando un aprendizaje mas profundo y activo, detectando las posibles carencias en su conocimiento, delimitando sus necesidades de aprendizaje y siendo capaces de obtener de forma autónoma y asimilar por sí mismos la información que necesitan.

Finalmente, se pretende conseguir que los alumnos aprendan a aplicar sus conocimientos académicos a la resolución de problemas, extiendan y profundicen los conocimientos adquiridos en las asignaturas, y que se produzca el afianzamiento conceptual de los contenidos explicados en las clases magistrales.

\section{Desarrollo de la innovación}

Para llevar a cabo el ABP 4x4 se utilizaron las sesiones reservadas para "grupos tutorizados" asignadas a las clases de Fisiología en el Grado en Medicina. Las asignaturas

(cc)) EY-NC-ND 2016, Universitat Politècnica de València

Congreso IN-RED (2016) 
elegidas corresponden al primer curso, primer semestre; y al segundo curso, primer y segundo semestre.

Son considerados créditos prácticos, y se evalúan hasta un $10 \%$ del $40 \%$ asignado a los contenidos prácticos en la evaluación de la asignatura.

Corresponden a 20 horas de docencia del profesor en un año académico, de las cuales cada alumno debe recibir 4 horas presenciales. Las 4 horas presenciales se dividen en 2 sesiones: una primera sesión preparatoria de 1 hora y una segunda sesión de resolución del caso de 3 horas de duración.

En la sesión preparatoria, que se realiza con la clase completa, se explica en qué consiste la metodología ABP, qué se evalúa, qué se espera del alumno, así como los objetivos que se han de alcanzar en la sesión presencial. Comenzamos de este modo con la primera fase del la metodología ABP 4x4 ("Análisis"). En esta fase de entrenamiento metodológico inicial se deben dar unas normas e instrucciones claras.

La siguiente fase ("Investigación") corre a cargo de los estudiantes, mediante trabajo autónomo del alumno (no presencial). Los alumnos pueden trabajan individualmente o en grupos, pero lo hacen sin intervención del profesor y fuera del horario de clases. Siempre pueden consultar dudas concertando una tutoría con el profesor o por correo electrónico.

Durante la sesión de 3 horas se llevará a cabo la fase de "Resolución" y la "Evaluación". En esta sesión se les convoca con suficiente tiempo de antelación y en grupos de no más de 16 alumnos.

Los casos de estudio se extrajeron de la base de datos del National Center for Case Study Teaching in Science, University at Buffalo. Uno de los principales objetivos de este Centro es desarrollar y mantener una colección nacional arbitrada accesible de estudios de casos ejemplares en todas las áreas de la ciencia para su uso en el pregrado, grado y nivel de secundaria. Este centro se dedica a recopilar casos de estudio para docencia desde hace más de 20 años y en su colección cuentan actualmente con más de 500 casos revisados (peerreview). La colección de los casos del NCSS ha sido citada por la Asociación Americana para el Avance de la Ciencia (AAAS) y el Consejo Nacional de Investigación (NRC) como fuente para casos de estudio modelo. Los casos son enviados por profesorado de, en su mayoría, facultades de ciencias de los EE.UU. y Canadá, aunque también hay autores que provienen de Suecia, Egipto y Australia.Todos los casos vienen acompañados de "apuntes o notas para el profesor" (teaching notes) y en su mayoría se acompañan también de un documento con la "clave de respuestas" (answer key).

\section{Resultados}

Durante 6 cursos académicos hemos realizado una adaptación de la metodología APB 4x4 en 3 asignaturas por año académico. Cada curso contó con una media de 86,8 estudiantes matriculados por cada grupo de clase. Los grupos para las sesiones presenciales constan de 9-16 alumnos (en promedio unos 12 alumnos/sesión). El total de alumnos a los que hemos 
enseñado mediante metodología $\mathrm{ABP}$ durante este periodo de tiempo es de 2700 estudiantes.

Las notas obtenidas fueron de media de 0,90 sobre 1 punto en total.

Como medida de la adquisición de competencias, se han comparado las notas obtenidas por los estudiantes que cursaron en sus grupos tutorizados $\mathrm{ABP}$, con los que realizaron otro

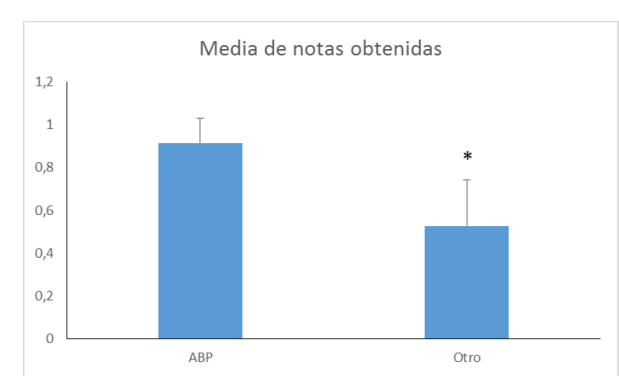

método. Para ello, hemos recopilado las notas de 1027 estudiantes, de los últimos 3 cursos académicos de 3 asignaturas diferentes. Como vemos en la gráfica adjunta la media de notas ha sido de 0,92 en los que cursaron ABP comparado con 0.55 en los que cursaron otro método de aprendizaje, siendo la nota máxima obtenible 1 punto.

Fig. 1 Media de las notas obtenidas en los grupos tutorizado realizando $A B P$ u otro métido. $n=1027$ estudiantes. $* p<0.01$

\section{Valoración de las actividades por los alumnos}

Se solicitó a los alumnos que habían concluido sus sesiones tutorizadas ABP que contestaran voluntariamente una serie de preguntas. Se recopilaron 140 encuestas. La valoración se realizó mediante un sencillo cuestionario con preguntas abiertas, en las que se preguntó:

1. ¿Consideras que has aumentado tus conocimientos tras la realización del caso de estudio?

2. ¿Consideras que estos conocimientos están dentro de los objetivos de aprendizaje de la asignatura?

3. ¿El tiempo invertido de trabajo está acorde a la puntuación que obtienes (hasta 1 punto del total de la nota final de la asignatura)?

4. ¿Te gustaría que se aplicase esta metodología docente en otras asignaturas?

5. ¿Recomendarías esta metodología docente a otro compañero/a?

6. Qué es lo que más te ha gustado de los casos de estudio ABP y lo que más te ha costado.

A la primera pregunta, el $99 \%$ de los estudiantes consideran que han aumentado sus conocimientos tras la realización del caso de estudio. Asimismo, el 98\% de los alumnos creen que los casos han sido bien seleccionados para encajar con los objetivos del curso (pregunta 2). Esto no es de extrañar, ya que se eligen siempre casos de estudio relacionados 
con los contenidos del temario teórico y que permitan ampliar los conocimientos aprendidos en el aula.

En cuanto a la tercera pregunta, el $65 \%$ por ciento de los estudiantes consideran que la valoración es escasa respecto al trabajo que han realizado. Les gustaría que aumentase el porcentaje o contribución del caso de estudio en la evaluación final de la asignatura. En la pregunta 4 , el 97\% de los encuestados contestan que sí les gustaría que se implementase esta metodología docente en otras asignaturas.

El 95\% de los alumnos recomendarían estas clases a otros compañeros.

Finalmente, de las 140 encuestas 30 contestan que lo que menos les ha gustado es la actuación del tutor; 79 contestan que lo que más trabajo les ha costado es encontrar la información necesaria para resolver la actividad; 25 contestan que la evaluación de la actividad contribuye con escaso porcentaje para la nota final, y 6 contestan que les ha gustado toda la actividad en su conjunto.

En cuanto a lo que más valoran los alumnos que han pasado por la actividad, el $46 \%$ contestan que les ha aclarado, aumentado o ayudado con los conceptos teóricos de la asignatura, un $16 \%$ contestan que su aplicabilidad en la práctica de la medicina, el $25 \%$ contestan que es una clase más entretenida o divertida, y el resto se dividen entre que lo que más les ha gustado es el papel del profesor o la facilidad para obtener la máxima puntuación de 1 punto en la evaluación de la actividad.

Una de las principales críticas por parte de los estudiantes está relacionada con la actuación del tutor. Probablemente, no todos los docentes a los que se les propone la realización de ABP estén familiarizados con la forma de proceder. Proponemos realizar una preparación a los profesores a los que se les ha asignado esa docencia, con unas bases de cómo se tutoriza un grupo de ABP.

Otra crítica que realizan los estudiantes es la dificultad para encontrar información. Si el tutor da unas buenas directrices a los alumnos de cómo buscar información, dónde, y de cómo se deben seleccionar y filtrar los resultados obtenidos, creemos que se solventaría el problema.

Finalmente, expresan que tiene poco peso la nota de la actividad en la nota total de la materia. Para realizar cambios en los porcentajes de evaluación se debería realizar el ABP en todos los grupos de la materia, ya que la evaluzación de una asignatura debe ser exactamente la misma para todos.

\section{Valoración de las actividades por los profesores}

Los compañeros docentes que han intervenido o participado directamente implementando esta metodología encuentran un alto grado de satisfacción en la aplicación del método. Sobre todo observan un alto grado de aprendizaje de los estudiantes, una mayor profundidad en la asimilación de los contenidos, la satisfacción que los alumnos expresan al concluir la clase presencial en el grupo tutorizado, y la positiva actitud de colaboración y participación. Además, todos coinciden en que se consigue una mayor implicación en la intensidad del trabajo, participación e interés, muy superior al observado en las clases

(cc) EY-NC-ND 2016, Universitat Politècnica de València

Congreso In-Red (2016) 
prácticas de laboratorio, las simulaciones por computador en el aula de informática, las tutorías o las clases magistrales en el aula.

\section{Conclusiones}

El ABP es un método aplicable y factible en grupos grandes en el limitado tiempo asignado para nuestras sesiones de grupos tutorizados.

Los alumnos expresan un elevado grado de satisfacción en las encuestas de opinión realizadas sobre las actividades en los grupos tutorizados.

Los profesores que desarrollan su docencia mediante metodología $\mathrm{ABP}$ en nuestros grupos tutorizados perciben una mayor implicación y trabajo del alumno respecto a otras actividades docentes desarrolladas en nuestro departamento como las prácticas de laboratorio, simulaciones por computador o las clases magistrales.

\section{Referencias}

1. Albanese, M.A. y Mitchell S. (1993). Problem-based learning: a review of literature on its outcomes and implementation issues, Acad Med 68: 52-81

2. Allen, D.E. y Duch, B.J. (Eds.) (1998). Thinking towards Solutions: Problem-based Learning Activities for General Biology. Philadelphia US: Saunders College Publishing ASHE-ERIC Higher Education Report No. 1, Washigton DC: The George Whasington University.

3. Barrows, H.S. y Tamblyn, R.N. (1980). Problem-Based Learning: An Approach to Medical Education, New York US: Springer.

4. Aspy, D.N., Aspy, C.B. y Quimby, P.M. (1993). What doctors can teach teachers about problem-based learning. Educational Leadership, 50, 22-24.

5. Branda, L.A. (2001). Innovaciones Educativas en Enfermería. El aprendizaje Basado en Problemas, centrado en el estudiante y en grupos pequeños. Rev ROL Enf 24(4):309-12.

6. Duch J., Groh S.E., Allen D.E. (Eds) (2001) The power of problem-based learning: A practical "how to" for teaching undergraduate courses in any discipline. Sterling Virginia US. Stylus Publishing. Sterling Virginia US

7. Font AR (2004)Líneas maestras del Aprendizaje por Problemas Revista Interuniversitaria de Formación del Profesorado. 18(1), 79-95.

8. Kaufman, A., Mennin, S., Waterman, R., Duban, S., Hansbarger, C., Silverblatt, H., Obenshain, S. S., Kantrowitz, M. Becker, T., Samet, J, \&Wiese, W. (1989). The New Mexico experiment: educational innovation and institutional change. Academic Medicine 64, 285-294.

9. Molina, J. A., García, A., Pedraz, A. y Antón, M. V. (2003). Aprendizaje basado en problemas: Una alternativa al método tradicional. Revista de la Red Estatal de Docencia Universitaria, 3 (2), 79- 85.

10. Newman MJ. Problem Based Learning: an introduction and overview of the key features of the approach. J Vet Med Educ. 2005 Spring;32(1):12-20.

(cc) BY-NC-ND 2016, Universitat Politècnica de València 
11. Norman GR, Schmidt HG. Effectiveness of problem-based learning curricula: theory, practice and paper darts. Med Educ. 2000 Sep;34(9):721-8.

12. Prieto, Barbarroja, Reyes, Monserrat, Díaz, Villarroel y Álvarez / Aula Abierta, 87 (2006) 171-194 UN NUEVO MODELO DE APRENDIZAJE BASADO EN PROBLEMAS, EL $A B P 4 X 4$

13. Schwartz P., Mennin S., Graham Webb (Eds.) (2001) Problem-based learning case studies, experience and practice. London UK KoganPage

14. Sulaiman F (2010) Students' Perceptions of Implementing Problem-Based Learning in a Physics Course. Procedia Social and Behavioral Sciences 7(C) 355-362. 